\title{
Fragilidad urbana: una lectura del desastre desde los habitantes menos protegidos
}

\author{
Urban fragility: a reading of the disaster from the least protected \\ inhabitants
}

Carlos Ríos-Llamas*

Recibido: julio 07 de 2019

Aceptado: noviembre 21 de 2019

\begin{abstract}
Resumen
Las catástrofes producidas por fenómenos llamados "naturales" obligan a pensar en la fragilidad de las ciudades y la vulnerabilidad de sus habitantes. Por un lado, el incremento exponencial de la urbanización y la concentración demográfica en las ciudades no solamente eleva el número de víctimas, sino que pone en evidencia la insuficiencia de la infraestructura urbana para la atención de los desastres. Por otro lado, el desastre suele presentarse de forma localizada, de manera que afecta más en las zonas urbanas donde los habitantes carecen de respuestas en términos económicos, sociales y de protección física. En este documento se reconstruyen los conceptos de fragilidad urbana y vulnerabilidad social desde un análisis general de la vivienda social en Guadalajara, los asentamientos irregulares en las periferias y la multiplicación de fraccionamientos populares que enfrentan las inundaciones y derrumbes desde la miseria cotidiana y la débil protección de las políticas urbanas. Se analizan cuatro componentes fundamentales para entender la fragilidad urbana y la vulnerabilidad social de forma conjunta: la exposición, las amenazas, las resistencias y las consecuencias. El objetivo principal es cuestionar al urbanismo contemporáneo desde la noción de fragilidad socioespacial, para evidenciar las lógicas mercantiles que se ocultan en la gestión del territorio. Desde esta perspectiva, los mal llamados desastres naturales, que son socialmente construidos y políticamente localizados, ponen al descubierto los mecanismos de polarización social y eliminación de los habitantes urbanos más vulnerables.
\end{abstract}

Palabras clave: fragilidad urbana, vulnerabilidad, riesgo, fraccionamiento popular.

\begin{abstract}
The catastrophes produced by the so-called natural phenomena force us to think about the fragility of cities and the vulnerability of their residents. On the one hand, the increase in urbanization and the demographic concentration in the cities not only increase the number of victims but also highlight the inadequacy of the urban infrastructure to deal with disaster. On the other hand, disaster usually occurs in specific circumstances, and it affects more in urban areas where the inhabitants already suffer lack of economic, social and physical protection. The notions of fragility and vulnerability are reconstructed from a general analysis of social housing in Guadalajara and informal housing projects suffering from summer floods, daily misery and the low quality of urban policies. The analysis is organized into four main components to understand urban fragility and social vulnerability together: exposure, threats, resistances and consequences. This document aims to question the urbanism from the notion of spatial fragility, to highlight the neoliberal mechanisms which are hidden in the contemporary discourses of the urban management. From this perspective, natural disasters highlight the mechanisms of social polarization and elimination of the most fragile urban inhabitants.
\end{abstract}

Keywords: fragility, vulnerability, risk, poor housing.

* Universidad De La Salle Bajío, México. Correo electrónico: carlosrios@delasalle.edu.mx 


\section{Introducción}

La ciudad de Guadalajara, en México, sigue siendo una de las más susceptibles en el país frente a los desastres ocasionados por lluvias en la temporada de verano. Por poner algunos ejemplos, el 10 de junio de 2018, las lluvias provocaron la inundación de calles, avenidas, plazas comerciales y algunas estaciones del tren ligero, poniendo en riesgo la vida de varias personas arrastradas por la corriente o encapsuladas en los vagones del tren; 1 de manera más reciente, el 30 de junio de 2019, varias zonas de la ciudad sufrieron daños considerables tras una tormenta con granizo que afectó varias calles, viviendas y vehículos. ${ }^{2}$ A pesar de que los gobiernos de los distintos municipios han incrementado sus estrategias para prevenir estos problemas, los procesos de urbanización del área metropolitana de Guadalajara parecen rebasar las acciones preventivas contra las inundaciones, sobre todo después de que en las últimas décadas se edificaron fraccionamientos residenciales en zonas periféricas sin respetar los procesos legales tanto en términos de impacto ambiental como en la calidad de la infraestructura y control de los riesgos.

Haciendo un ejercicio en retrospectiva, desde el caso concreto de la colonia Lomas del Sur, se puede verificar cómo en los primeros años, posteriores al 2000, apareció un fraccionamiento popular, de la noche a la mañana, entre la zona natural que separaba al poblado de Tlajomulco de la mancha urbana de Guadalajara. Como Lomas del Sur, que aquí nos servirá como caso de estudio, otros fraccionamientos populares emergieron en las periferias urbanas con el principal objetivo de mercantilizar el territorio desde varias inmobiliarias. Rebasando las responsabilidades compartidas entre desarrolladores urbanos, instituciones de crédito a la vivienda y gobiernos locales, los problemas que aumentan la vulnerabilidad de la población frente a los desastres como inundaciones y derrumbes no fueron considerados como un elemento central del desarrollo urbano, sino que se fueron dejando como una responsabilidad que deberían atender los siguientes responsables de gestionar estos espacios.

Los desastres "naturales" han sido conceptualizados como un resultado incontrolable de las fuerzas de la naturaleza. En este sentido, tanto las inundaciones como los terremotos y otra suerte de eventos se piensan como inevitables, tanto en su origen como en sus consecuencias. Sin embargo, el desastre no tiene únicamente un componente azaroso e imprevisible, sino que pone en evidencia la fragilidad de los sistemas de protección que ha reproducido el ser humano.

\footnotetext{
${ }^{1}$ El periódico Excélsior, bajo el título “Tormenta provoca inundaciones en calles, plaza y tren ligero de Guadalajara”, indicaba que, debido a las inundaciones, los bomberos rescataron a una persona que, atrapada en su auto, fue arrastrada por la corriente, y a otra persona que sufría de hipotermia luego de la inundación de los vagones del tren ligero.

${ }^{2}$ De acuerdo con Torres (2019), del periódico El Universal, se reportaron daños en 457 casas y 80 automóviles de varias zonas de la conurbación de Guadalajara.
} 
Muchas de estas técnicas y formas de responder a un fenómeno natural dependen de las condiciones sociales en las que se desenvuelven los habitantes en su vida ordinaria. Robert Castel, en sus estudios sobre el riesgo, puso mayor atención en los sistemas de protección social que en la vulnerabilidad (Martin, 2013). Castel (2006), en su obra La inseguridad social ¿Qué es estar protegido?, da la vuelta a las clásicas explicaciones de las desigualdades desde la producción social del riesgo y propone un análisis a partir de los sistemas de protección.

En efecto, mientras que las explicaciones clásicas de las catástrofes suelen poner el acento en el riesgo que experimentan los emplazamientos o las sociedades, la vulnerabilidad de los habitantes frente a los desastres también puede entenderse desde la capacidad de respuesta de las estructuras sociales frente al peligro que representan los constantes desajustes y reajustes de la naturaleza. La fragilidad de las redes de protección social, entonces, es esencial para entender el desastre, producido no tanto por un evento de la naturaleza sino por la poca capacidad de algunas sociedades para hacer frente a lo imprevisible.

Vista desde lo urbano, la fragilidad estaría relacionada con las dinámicas socioespaciales que se materializan en la ciudad en forma de espacios excluyentes o integraciones desiguales (Wacquant, 2007; Bayón, 2015). Si bien no se puede negar que las desigualdades sociales tienen una implicación espacial y un referente sobre el territorio, la mayoría de los estudios realizados sobre las disimetrías sociales están concentrados en análisis estadísticos de ingresos y otros componentes socioeconómicos. Conviene, como una aportación de los estudios urbanos que aporten una lectura espacial de los fenómenos, profundizar en los procesos y mecanismos de configuración socioespacial que derivan en la expresión de injusticias en las ciudades.

De acuerdo con el análisis de Young (1990) sobre la injusticia, ésta no debe ser vista desde los modelos de distribución de la riqueza, los ingresos u otros bienes materiales, sino desde aspectos como la cultura, la toma de decisiones y la distribución del trabajo, y es necesario tomar en cuenta otras dimensiones como la política, la organización institucional, la acción pública, las prácticas y los hábitos sociales y la producción cultural de significados. Como consecuencia, Young entiende la injusticia como un resultado de dos condiciones sociales: la dominación y la opresión.

La dominación se refiere a las condiciones institucionales que inhiben a la población para participar en la determinación de sus propias acciones, o las condiciones de sus actos. La opresión, por su parte, se relaciona con la inhibición de algunos grupos sociales para desarrollar y ejercer sus capacidades y expresar sus propias necesidades, pensamientos y sentimientos. En la lógica de la misma autora, la opresión puede tomar cinco formas combinables: 
1) La explotación: ligada al sistema capitalista y a la pérdida del reconocimiento colectivo

2) La marginalización: exclusión del empleo y de la vida social.

3) La ausencia de poder: exclusión en la toma de decisiones.

4) El imperialismo cultural: ligado a la invisibilidad de un grupo y la universalización de formas culturales del grupo dominante.

5) La violencia colectiva: que se convierte en una práctica social aceptable (Young, 1990:37-63).

Otros autores, como Rawls (1971) y Sen (1995), han ampliado el concepto de justicia; el primero ha insistido en la constante negociación de las sociedades para decidir sobre lo que es justo e injusto; y el segundo ha enfatizado en aclarar que las condiciones sociohistóricas y los determinantes demográficos juegan un papel fundamental para entender lo que es justo para un individuo o para un grupo social. En este sentido, no existe un principio inamovible de lo justo para diferentes tiempos ni para diferentes sociedades, sino que debe analizarse desde las mismas construcciones y valores epocales y culturales de los individuos y sus colectividades.

La justicia espacial, como una lectura alternativa de la gestión del territorio desde la geografía y el urbanismo, se inscribe como una oportunidad de análisis que permite el diálogo entre el territorio y los asuntos sociales. Varios autores, como Harvey (2008) y Musset (2010), acercan los planteamientos teóricos sobre la desigualdad a sus componentes urbanos y su materialización a lo espacio-temporal de las ciudades. Siguiendo esta propuesta, se pueden plantear al mismo tiempo dos preguntas: sobre los procesos que producen la segregación socioespacial y sobre la injusticia que se materializa en la distribución desigual de los recursos y los riesgos.

En este sentido, la justicia se podría convertir en un referente para la mayoría de las políticas que impactan sobre el territorio; de la misma manera, todos los procesos de urbanización se podrían orientar hacia la búsqueda por la equidad socioespacial. Los entornos de fragilidad urbana, en esta óptica, son una muestra de la desigualdad en la distribución de los riesgos y los determinantes sociales en las ciudades. La fragilidad urbana se expresa también en la fragilidad de los cuerpos y la eliminación "natural" de los habitantes más débiles como resultado del fracaso de propuestas de protección y movilidad social en los modelos actuales de urbanización. En efecto, la emergencia de fraccionamientos populares en las zonas periurbanas, en medio de tantas irregularidades con respecto a la gestión de los riesgos, obedece tanto a la mercantilización del territorio, derivada de la acumulación de capital a partir de la urbanización, como a la injusta distribución de los recursos entre los habitantes. 
Las nociones de vulnerabilidad y de justicia espacial tiene diversidad de abordajes, los cuales se pueden integrar de acuerdo con los estudios de las desigualdades urbanas; es decir, desde la noción de Moser (1998) sobre la vulnerabilidad, entendida desde la capacidad de los individuos para sobreponerse a los riesgos, hasta la conceptualización de Bourdin (2016) sobre la ciudad frágil por la inestabilidad de las políticas urbanas. Los estudios que vinculan los riesgos ambientales con las configuraciones territoriales no siempre articulan la dimensión material del territorio con el tejido social que lo constituye.

Como propuesta de este artículo, y desde el enfoque de los riesgos, la vulnerabilidad y sus componentes de fragilidad urbana, injusticia espacial y desigualdades sociales, se dirigen a la explicación del porqué de la existencia de una mayor exposición de algunos territorios y sus habitantes frente a las catástrofes. Además, se considera necesario analizar, más allá de las condicionantes demográficas y los factores físicos del territorio, la organización social y la manera como los grupos humanos responden a las eventualidades de la naturaleza.

Si los desastres no ocurren en todos los lugares ni tienen la misma intensidad, es precisamente porque más allá de la irrupción de un evento catastrófico está el fracaso de sus estructuras de protección. Así, no es lo mismo un temblor o una inundación en una zona habitacional de élite, al impacto que tiene cualquiera de estas eventualidades en los asentamientos irregulares o en las zonas de vivienda social. Entonces, no todos los riesgos terminan en desastre y no todos los grupos sociales los sufren de la misma manera.

Los procesos sociales generan una exposición desigual frente a los riesgos. Algunos habitantes de las ciudades serán más frágiles frente a los peligros de esta suerte de eventos. Un elemento fundamental, además de la organización social y del capital cultural de las personas, es la condición física tanto del entorno donde habitan como de su propio cuerpo. Desde este ángulo, la fragilidad del espacio puede volverse exponencial en la fragilidad de un cuerpo humano más débil, enfermo o con salud deteriorada, cuya exposición al riesgo sería mayor que la de un cuerpo adulto promedio.

Las relaciones sociales, por otro lado, implican también un cierto grado de protección o de inseguridad frente a los fenómenos naturales. En un enfoque más social, como el que se pretende en esta conceptualización, la mitigación de los riesgos implicaría un trabajo concentrado en la multiplicación de estrategias para reducir las fragilidades urbanas, es decir, una serie de transformaciones de carácter sociopolítico y socioeconómico que irían desde el combate a la precariedad urbana hasta el refuerzo de los sistemas de protección y promoción de redes de organización y participación social. 
Desde este enfoque, la fragilidad, al igual que la vulnerabilidad, tendría dos ángulos: "una cara exterior del riesgo [...] a la que todos los individuos están sujetos, y una cara interior que sería la indefensión entendida como la falta de recursos para resistir y sobreponerse a las pérdidas" (Schröder-Butterfill y Marianti, 2006). Desde esta perspectiva, la comprensión de la fragilidad y las metodologías para medirla implicarían la construcción de una serie de indicadores sobre las estrategias de prevención del desastre y la posibilidad de implementarlas. Entonces, la "vulnerabilidad frente al desastre" es resultado de un proceso continuado de fragilización en el que se inhiben o debilitan las posibilidades de hacer frente a una eventualidad que no debería convertirse en una catástrofe.

\section{La fragilidad y vulnerabilidad analizadas desde el enfoque espacial del desastre}

Las catástrofes mucho tiempo fueron consideradas como un resultado directo de los fenómenos naturales, como inundaciones, terremotos y sequías. Más tarde, esta mirada se vio rebasada al constatar que no todos los riesgos concluyen en desastres y que no todas las personas afectadas reaccionaban de la misma manera. De acuerdo con SchröderButterfill y Marianti (2006:10), la noción de vulnerabilidad es la que mejor ayuda para entender los vínculos entre el riesgo, valorado como una amenaza que proviene del exterior y las formas de respuesta de los grupos sociales.

Por otra parte, la mayor parte de la literatura anglófona sobre la vulnerabilidad se concentra en sus componentes económicos, como los niveles de ingreso; por tanto, la vulnerabilidad se relaciona de manera cercana con la pobreza; más aún, cuando se toman en cuenta factores de carácter espacial, como la vivienda; se considera que una persona es vulnerable si sus ingresos son bajos, si su vivienda es de poca calidad o si no tiene acceso a los sistemas de protección social (Martin, 2013).

Para otros autores, como Chambers, la vulnerabilidad es un concepto de carácter "probabilista" determinado por la relación de proximidad entre un sujeto y un daño (citado por Schröder-Butterfill \& Marianti, 2006). Desde esta comprensión, se podría entender de manera cuantificable como la resultante acumulada de un conjunto de riesgos que se materializan en el espacio construido. Como señala Sen (1995), si la diversidad de condiciones y circunstancias de los individuos y sociedades es fundamental para entender lo que les conviene, la fragilidad urbana se presenta como una alternativa para la lectura localizada de los desastres y los determinantes socioespaciales que condicionan las posibilidades de respuesta de cada colectivo.

Entonces, la fragilidad urbana puede entenderse desde la capacidad de las ciudades para hacer frente al riesgo por medio de tres elementos principales: la constitución demográfica, la organización del espacio y las formas urbanas. Los factores sociodemográficos se manifiestan en la configuración de las estructuras sociales y la 
calidad de los vínculos entre los individuos; la organización del espacio se refleja en las características que determinan la calidad de la infraestructura y de los servicios públicos; $\mathrm{y}$, finalmente, la configuración de los espacios se refiere al papel que desempeñan las formas urbanas para sostener o transformar las dinámicas de la vida ordinaria de los habitantes.

En los últimos años, algunos estudiosos de la antropología han propuesto la ciudad como uno de sus objetos propios. Por ejemplo, para Agier (2015), la etnografía urbana es una manera de producir descripciones e interpretaciones sobre la ciudad a partir del registro continuado de observaciones, datos inductivos y reflexiones del investigador. De acuerdo con el autor, el abordaje antropológico de lo urbano permite pensar en la ciudad desde los habitantes, sus relaciones e interacciones y las lógicas sociales, políticas o culturales que se despliegan en las urbes. Otros autores proponen la reconstrucción, incluso gráfica, de los espacios, recorridos, trayectorias y experiencias del investigador en los escenarios de estudio (KOSMOS, 2018).

Se trata de romper con la idea de una antropología clásica, la cual se concentraba en el estudio de poblaciones originarias alejadas de las concentraciones urbanas, en donde el antropólogo documentaba una serie de datos de una cultura que se le presentaba como ajena. La perspectiva y los procedimientos propuestos en este análisis retoman la idea de que lo urbano se reconstruye a los ojos del investigador y desde sus propias reflexiones. Las observaciones del espacio, las interacciones y los diálogos con los habitantes son posteriormente reconstruidos en un ejercicio crítico desde la óptica concreta de la fragilidad urbana.

A diferencia de la sociología urbana, en el trabajo antropológico no existe un código para la producción de entrevistas mediante el que se analiza el texto (Althabe, 2008), sino que existe un proceso de codificación y análisis en el cual el investigador está inmerso. En lugar de tomar distancia del objeto de estudio, el antropólogo es un observador que forma parte de la observación misma, con lo que se evita la tendencia al objetivismo del caso de estudio.

El escenario específico para el estudio es el fraccionamiento popular de Lomas del Sur, en el municipio de Tlajomulco, en el sur de Guadalajara, cuyo crecimiento exponencial en términos del aumento de proyectos de vivienda social se ha visto involucrado desde su origen en una serie de deterioros ambientales, corrupciones políticas con respecto a la calidad de la vivienda y la débil articulación de la vida ordinaria con los servicios públicos con que deberían contar los habitantes de la zona. 
Con respaldo en la propuesta de Schröder-Butterfill y Marianti (2006), se considera que un análisis de la exposición, las amenazas, los vínculos sociales y las consecuencias ayudarían para construir una perspectiva socioespacial de los riesgos y de la fragilidad de los sistemas urbanos. Para ilustrar esta propuesta desde un caso de estudio y un abordaje antropológico del problema del riesgo, se analiza una catástrofe: las inundaciones ocurridas en varios fraccionamientos populares del sur de Guadalajara en 2016. A partir de esta aparente eventualidad que tiene cierta persistencia cada temporal de lluvias, se establece un análisis de los componentes de la fragilidad urbana y la vulnerabilidad de los habitantes en la zona.

Durante 2015 y 2017 se realizó un trabajo de campo en Lomas del Sur que implicó avecinamiento en el fraccionamiento popular, observación participante durante varios meses y aplicación de entrevistas a los habitantes. El registro de los datos se hizo a partir de diario de campo, grabaciones y archivo fotográfico. El análisis posterior se organizó en dos partes: la primera describe los procesos de precarización y la segunda muestra la intervención antropológica a partir del diálogo entre la teoría y las notas de campo. Este artículo se apoya en ese estudio y se concentra específicamente en los procesos de precarización social y en las situaciones de desastre.

A partir de un ejercicio de conceptualización desde la teoría crítica, se rescatan las narrativas del trabajo de observación y el diario de campo del investigador para reflexionar sobre la manera de construir los conceptos de riesgo, vulnerabilidad y desastre. Las narrativas manifiestan el acercamiento a la vida ordinaria de los habitantes de Lomas del Sur, en la periferia de Guadalajara. De manera simultánea al ejercicio crítico, se establece una serie de reflexiones sobre la fragilidad urbana, la vulnerabilidad social y su expresión en el desastre desde las implicaciones del urbanismo en torno a cuatro ejes de análisis: la exposición, las amenazas, las resistencias y las consecuencias.

\section{Exposición: la fragilidad del entorno construido}

La colonia Lomas del Sur es uno de los fraccionamientos populares resultado del crecimiento descontrolado de Guadalajara en la primera década del siglo XXI. Aunque las primeas construcciones de viviendas datan de 2002, la preventa de las casas implicó que muchas personas comenzaran a pagar el crédito inmobiliario sin haber visto la mínima tarea de construcción. De hecho, algunos vecinos recuerdan cómo la primera visita al sitio era simplemente para indicar, en medio de matorrales y estancamientos lodosos, dónde sería su buena casa. Comprar en preventa, cuando la inmobiliaria no ha tocado físicamente el sitio, aseguraba un costo casi por la mitad de los precios de viviendas terminadas. 
El problema de encharcamientos y nacimientos de agua es anterior a la construcción de casas en Lomas del Sur (ver mapa 1). Algunos trabajadores de la constructora, y que hoy viven en el fraccionamiento porque consiguieron facilidades de pago, recuerdan cómo se colaban las cimentaciones sobre nacimientos de agua. Los problemas que surgieron en más de la mitad de las casas a raíz de la humedad y los agrietamientos, sumados a la falta de infraestructura, hicieron que más del $40 \%$ de los habitantes abandonara sus casas y perdiera sus créditos porque tenía que elegir entre pagar la mensualidad de la vivienda o reparar sus problemas (Ríos, 2018).

Mapa 1. Lomas del Sur y el modelo geohidrológico integral de las cuencas de Atemajac y Toluquilla.

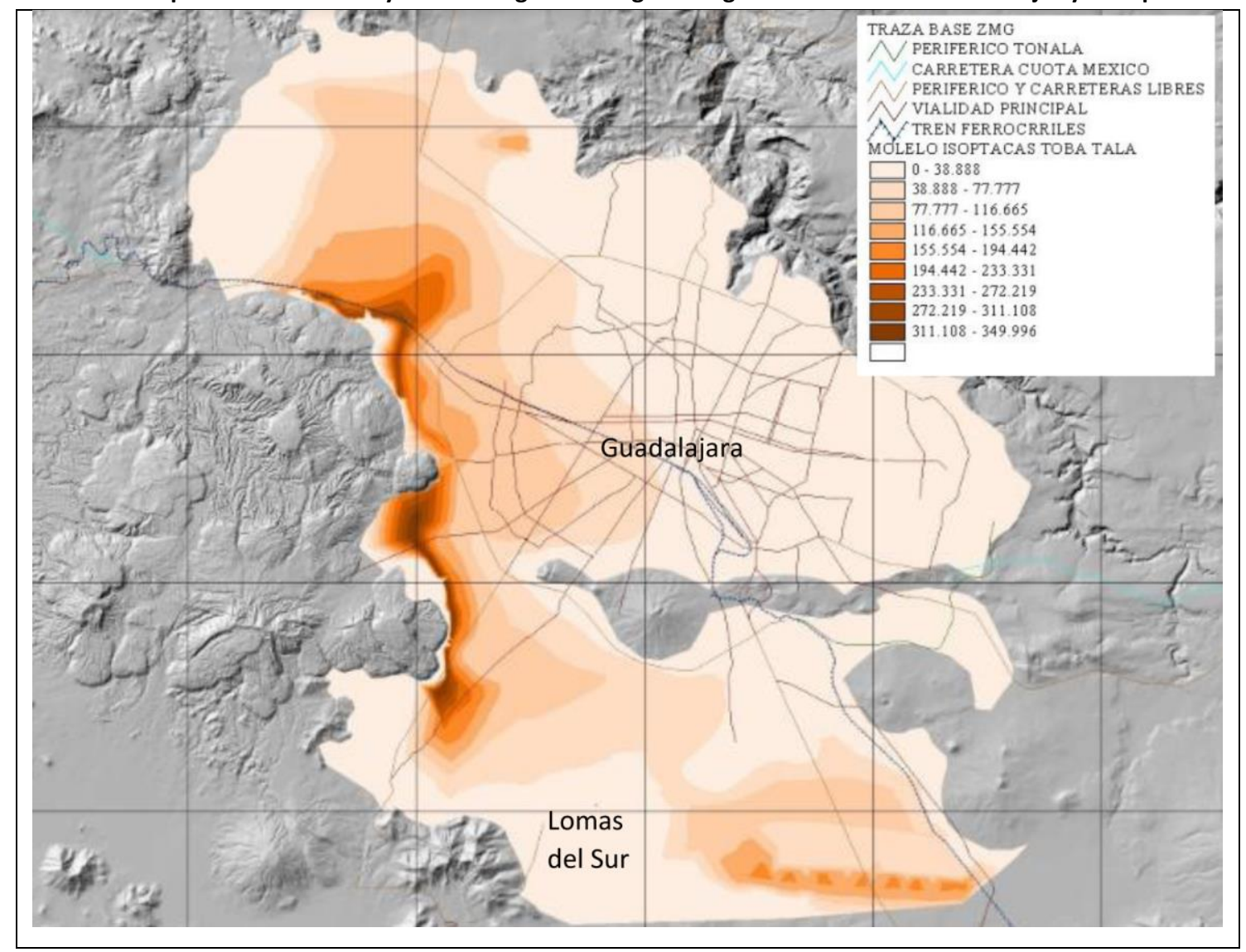

Fuente: Extracto del Modelo Isopacas Toba Tala. Siapa

Esto explica cómo, una década después, algunos vecinos, como Don Pedro, ${ }^{3}$ siguen año con año reparando los rincones de su casa por donde resurge la humedad. Don Pedro habita en Lomas del Sur desde que se construyó el fraccionamiento; vive en una casa con constantes fugas de agua y humedades en cada rincón de sus muros, así como goteras en la azotea. La casa, construida en una sola planta como todas las del fraccionamiento,

${ }^{3}$ Todos los nombres de los habitantes son ficticios a fin de salvaguardar la identidad de los informantes. 
incluye un espacio de sala-cocina-comedor, una recámara, una pieza con WC y ducha y un área de lavado. Al frente de las viviendas se cuenta con un espacio para cochera, pero Don Pedro lo modificó para tener una segunda recámara, donde se instaló con su esposa; la otra habitación la dejó a sus dos hijas; una de ellas es madre soltera de un niño de no más de cinco años.

Don Pedro trabaja para la empresa de tecnología IBM, en Guadalajara. Para llegar a su trabajo, todos los días debe desplazarse hasta la ciudad en su auto, el cual casi siempre le ocasiona problemas que requieren reparación. Para reducir el gasto en la gasolina, se organiza con tres de sus vecinos varones que habitan en la misma calle, a fin de aminorar los costos y llegar a buena hora hasta su trabajo. De otra manera, si utilizaran el transporte público, tendrían que salir de sus casas a las 4:30 am por el hacinamiento en las unidades del transporte y el tráfico al ingresar a la zona interior de Guadalajara, que puede variar entre los 90 y los 150 minutos.

Además, las políticas de empresas como IBM, amparadas con sistemas de outsourcing, ${ }^{4}$ no les otorgan días de descanso a sus empleados porque trabajan a partir de contratos cortos que se renuevan, en promedio, cada 4 meses. Con estas condiciones, no existe ninguna seguridad de ingreso económico para la familia que se describe más allá de estos periodos de contratos intermitentes. Además, el soporte de seguridad social se ve afectado por esta tercerización del empleo que opera a partir de subcontrataciones y que dificulta la constancia en los soportes de seguridad social.

María, la esposa de Don Pedro, repara ropa en su misma casa. No existe ninguna formalidad en este empleo, pero se sostiene gracias a la recomendación inmediata de sus vecinos y conocidos y en la organización de sus actividades domésticas y el tiempo que dedica a estas tareas eventuales. Entre los encargos, realiza zurcidos, remiendos o bastillas, que apenas alcanzan cincuenta pesos y esto no lo considera como un empleo. La economía de subsistencia también requiere un horizonte próspero para redituar, y en Lomas del Sur lo que se acumulan son capas de miseria en diferentes manifestaciones.

A sus más de sesenta años de edad, Don Pedro debe seguir trabajando para cubrir tres gastos esenciales: sostener a su familia, pagar el crédito de su casa y reparar constantemente los desperfectos de la vivienda y del auto. No acumula dinero para vacaciones; no le alcanza para eso. A veces tiene que decidirse entre pagar el crédito o reparar las salidas de humedad en el piso y en el techo de su casa para evitar el daño de los muebles. Por fortuna, como él considera, tiene un vehículo en qué moverse y eso le facilita el trabajo. El resto de la población de Lomas del Sur debe tomar camión y

\footnotetext{
${ }^{4}$ De acuerdo con el Colegio de Contadores Públicos de México (2016), el outsourcing o tercerización "es una figura cuyo objetivo es colaborar con las empresas en la realización de tareas y actividades, ya sean operativas o de soporte, que permite a éstas concentrarse en las actividades que sí dominan y, por ende, en el logro de sus objetivos, incrementando su productividad, eficiencia y crecimiento". El Colegio reconoce que esta figura ha sufrido abusos con respecto al pago de impuestos sobre el salario, la seguridad social de los trabajadores y la subcontratación de servicios laborales.
} 
transbordar hasta dos veces para ir a trabajar todos los días. Frente a los constantes problemas de falta de agua, y luego de inundaciones, la vida de Don Pedro y su familia es muy inestable.

No se puede suponer que sea el caso de todos los habitantes de los fraccionamientos populares, y ni siquiera de la colonia Lomas del Sur. Este nivel de exposición ante las eventualidades se construye desde situaciones específicas de configuración de los hogares y de frente a los problemas de un urbanismo deficiente y corrupto. La mayor exposición frente al desastre, como se puede observar, está articulada íntimamente con las dinámicas ordinarias de los habitantes y su poca capacidad de respuesta a cualquier eventualidad.

El enfoque de la vulnerabilidad, desde un análisis de las condiciones ordinarias en los espacios domésticos, "remite a un estado de los hogares que varía en relación inversa a su capacidad para controlar las fuerzas que modelan su propio destino, o para contrarrestar sus efectos sobre el bienestar" (Kaztman, 2000). La exposición frente al riesgo, entonces, está proporcionalmente dada en términos de las limitaciones que experimentan los miembros de cada familia en la solución de las necesidades más básicas. Para el caso específico de Lomas del Sur, así como el de muchos hogares en las zonas periféricas de ciudades latinoamericanas, la opresión económica aumenta la exposición de cada uno de los miembros frente a cualquier desajuste socioespacial que pudiera ocurrir por el desequilibrio en términos ambientales.

Desde un enfoque antropológico y el análisis crítico de la vida ordinaria, la exposición implica considerar que el espacio se organiza y funciona de acuerdo con la capacidad de acción de los habitantes retratada en lo más simple de su cotidianidad. Más que una lectura del momento fortuito de la catástrofe, se trata, entonces, de profundizar sobre las estructuras de seguridad y protección social que podrían reaccionar como una plataforma de soporte en el caso de cualquier fenómeno motivado por el reordenamiento de la naturaleza.

\section{Amenazas: las situaciones de riesgo}

El segundo elemento para comprender la fragilidad urbana frente al desastre es la construcción socioespacial de las amenazas. Tanto Beck (2008) como Castel (2006) consideran que la sociedad contemporánea enfrenta una serie de inseguridades porque ya no tienen un soporte en las estructuras sociales .Por un lado, los desajustes en el medio ambiente son una prueba de la incapacidad del hombre para controlar el mundo a partir de la ciencia y la tecnología; además, estos problemas exponen las limitaciones del hombre para prever soluciones seguras y estables. 
En la colonia Lomas del Sur, así como en varios fraccionamientos populares de Guadalajara, la capacidad de los habitantes para actuar frente a las inundaciones del verano es casi nula. No existen sistemas de prevención, no hay regulaciones para controlar los riesgos y ni siquiera se han vigilado las normativas básicas antes de construir las viviendas y circulaciones de la colonia. Por otro lado, la irregularidad de algunos asentamientos y la falta de vigilancia de las condiciones mínimas de las viviendas incrementan el riesgo en algunos hogares que se erigen y mantienen al margen de cualquier regulación municipal y estatal.

Este es el caso de algunas familias, como la de Lucía, que se describe brevemente en los siguientes párrafos. Ella habita en una ladrillera contigua a Lomas del Sur desde hace años. Sus tres hijos, una niña de secundaria y dos niños de 8 y 10 años, viven con ella en una humilde construcción de láminas, de una sola pieza, y con piso de tierra. Los cuatro trabajan: Lucía recicla cartones y botellas, su hija vende nopales casa por casa y los dos niños trabajan todo el día haciendo ladrillos. Su vivienda es de materiales que se fueron reciclando poco a poco. Un espacio multiusos, cubierto con restos de lámina, maderas y plásticos, sirve como cocina, salón y dormitorio. La vivienda no tiene piso y la puerta de madera se encuentra sobre puesta y que atada con un cordón para cerrarse.

El día 2 de octubre de 2016, el gobierno del municipio de Tlajomulco reportaba una situación de contingencia para muchas colonias que se habían inundado tras fuertes lluvias (Gobierno de Tlajomulco, 2016). Sin embargo, la inundación impacta de manera mucho más importante a familias como la de Lucía, cuyas viviendas y pertenencias se perdieron por completo. Su casa se inundó y todas sus posesiones fueron arrastradas por la corriente y revolcadas en el lodo. Las lluvias que persistieron por más de 48 horas afectaron a 10 colonias y fraccionamientos de manera grave, y a otros 13 con daños importantes. El reporte oficial indicaba que, además de las inundaciones, se presentaron varios derrumbes en la zona, con afectaciones significativas a las viviendas.

Como puede notarse, el caso de Lucía frente a esta catástrofe es radical: pérdida de vivienda y refugio con algunos amigos. Además de la fragilidad urbana y la débil protección que significan la infraestructura y servicios, la situación de Lucía y sus hijos frente a la crisis revela la mayor exposición de algunos individuos frente a catástrofes y la construcción socioespacial del riesgo que vulnera de manera localizada a los hogares de los márgenes. Si bien es cierto que cada habitante de Lomas del Sur enfrentó las inundaciones con estrategias propias y a partir de los recursos disponibles, no se puede estandarizar la eventualidad como si todos los que comparten una ubicación geográfica contaran con las mismas plataformas para hacer frente a las catástrofes. 
El caso de los habitantes de Lomas del Sur y la narrativa de uno de ellos no pretenden la generalización, sino la ilustración de las situaciones de riesgo a partir de las condiciones de precariedad dispuestas por el emplazamiento de la vivienda y por la estructura social y familiar. De hecho, la antropología, en primer lugar, no busca una lectura de estructuras y procesos sociales, sino la manera como estas estructuras y procesos significan y modelan la vida humana. Un habitante, una familia o un fraccionamiento no son una muestra que pueda superponerse en otros contextos, pero la reflexividad a que invita la antropología supone que tanto el habitante como la familia y el fraccionamiento son construcciones de sentido que corresponden a la manera como pensamos y edificamos el espacio habitable.

Desde la mayor exposición de algunos habitantes frente a las situaciones de riesgo y regresando a Beck (2008), podemos afirmar que la sociedad contemporánea ya no parece estar gobernada por el control y la seguridad, sino por el riesgo y la incertidumbre. Ante el estado político rebasado en términos de garantía del bienestar de los individuos, aparece la presión global que delimita las actuaciones de las instancias gubernamentales desde los requerimientos financieros y mercantiles en el orden internacional. Precisamente, en esta incertidumbre reposan las amenazas que vulneran a las zonas urbanas y a sus habitantes: la incapacidad de los seres humanos para asegurar el porvenir.

\section{Resistencias: los vínculos sociales}

El tercer elemento que aquí se propone para entender la fragilidad urbana y la vulnerabilidad socioespacial está relacionado con los vínculos sociales y su capacidad para resistir a las catástrofes. Para una comprensión más equilibrada de la fragilidad urbana, es importante considerar que la débil protección que aporta la ciudad se suele compensar por medio de los vínculos sociales que soportan a los seres humanos más frágiles. Aunque el costo suele ser elevado en términos de la calidad de vida, muchas mujeres de Lomas del Sur combinan el cuidado de los hijos, adultos y enfermos con las tareas domésticas y el trabajo nocturno.

Las redes de vecinos y los líderes de manzana son portavoces de los problemas de cada calle o clúster, ${ }^{5}$ como falta de agua, de luz eléctrica y de otros servicios que se interrumpen con frecuencia. En el caso de los fraccionamientos populares, la protección y el confinamiento que supone la configuración urbana de clústeres están casi siempre rebasados por falta de cuotas establecidas para el mantenimiento, la vigilancia y la organización social.

\footnotetext{
${ }^{5}$ Se entiende clúster como el grupo de viviendas de interés social que se integran en una unidad confinada, a veces con sistema de vigilancia o, al menos, con un portal que impide el acceso a estos espacios residenciales.
} 
Durante el verano de 2015, en el entorno del fraccionamiento Lomas del Sur se comenzaron a construir varios desarrollos inmobiliarios. Las obras de construcción de Las Trojes y Valle de los Encinos pronto ocasionaron desajustes en el abastecimiento de agua. ${ }^{6}$ Las licencias de urbanización de ambos fraccionamientos tienen fecha de agosto de 2014 (Gobierno de Tlajomulco, 2014), pero la gestión de los recursos como el agua no se resolvería sino de manera posterior. Como el prototipo de vivienda de Lomas del Sur no contaba con cisterna ni tinaco, los vecinos tenían que organizarse para pedir todos los días pipas al ayuntamiento municipal para los distintos clústeres. Este problema, que se siguió durante varias semanas de abril y mayo, estuvo repitiéndose con intermitencia el resto del año y hasta la regularización del servicio al año siguiente.

Con el pretexto del desabasto de agua, se organizaban reuniones de vecinos por las noches, cuando la mayoría había regresado de sus trabajos. Ahí se podían identificar varias dificultades: en primer lugar, casi todos los vecinos cuestionaban las acciones de su representante frente a las autoridades locales, mientras la líder de vecinos afirmaba estar en constante diálogo con el municipio para pedir pipas con agua, los vecinos insistían en la falta de garantías con respecto al día y la hora en que se enviaba el agua, lo que según ellos implicaba una falta de carácter y autoridad que les garantizara recibir el servicio. Al mismo tiempo, los vecinos coincidían en que la eventualidad del desabasto se debía a errores de las empresas constructoras y que la representante no había establecido ningún canal directo para comunicarse con los empresarios y exigirles control sobre el uso del agua pública.

Por otro lado, en estas reuniones, organizadas en medio de la calle, apenas se podía contar con tres o cuatro varones entre una veintena de mujeres. Ellas culpaban la falta de voces masculinas para hacerse escuchar frente a las autoridades y explicaban cómo cada que se organizaba una comitiva para ir a reclamar en las oficinas nunca había ningún hombre; el argumento, decía una de ellas, era que "cuando ven a puras viejas, nadie les hace caso".

Poco después, sobrevinieron las inundaciones en Lomas del Sur al verano siguiente, ante lo cual no fue sorprendente la débil organización vecinal para hacer frente al desastre y emitir un discurso claro frente a los actores gubernamentales y empresariales implicados. Las redes de vecinos guardaron silencio y esperaron a que los representantes de gobierno actuaran: víveres, limpieza de las calles, reparación de la infraestructura eléctrica y otros detalles menores.

\footnotetext{
${ }^{6}$ En el municipio de Tlajomulco, la dotación de los servicios de agua potable se realiza a través de la perforación de pozos profundos y es administrada por la Dirección de Agua Potable y Alcantarillado, dependiente del Ayuntamiento de Tlajomulco de Zúñiga.
} 
Al final, la inundación puso en evidencia que los vínculos sociales no constituían una plataforma de protección, reacción y prevención de circunstancias, como la que se había presentado. El descrédito de unos vecinos contra los otros había logrado una fragmentación social que se expresaba en la búsqueda de culpables entre los mismos habitantes. Las acciones emprendidas por la municipalidad, cualquiera que fueran, no se seguían a una exigencia de los vecinos, sino a la voluntad de los gobernantes.

El estudio de la fragilidad urbana no sólo debe poner atención en las condiciones físicas del entorno construido y en las características fenomenológicas de las amenazas y riesgos, sino en las maneras de organización de los individuos para movilizar los recursos materiales y sociales como estrategia para hacer frente a los desastres. Retomando a Castel, muchas veces "los individuos no son capaces de resistir por sí solos", les hacen falta apoyos y protecciones que aseguren su futuro y el de sus seres queridos" (citado en Martin, 2013).

Desde esta perspectiva, indica Martin (2013), la vulnerabilidad puede pensarse desde una doble fragilización: la del empleo con el que se asegura la obtención de recursos materiales, y la de los vínculos sociales relacionados con la inestabilidad de las familias. El problema de los espacios como Lomas del Sur es que los fraccionamientos populares suelen integrarse a partir del avecinamiento de individuos que llegan sin conocerse y que muchas veces no alcanzan una buena integración por múltiples desavenencias socioculturales. Además, la desorganización social se ve reforzada con la emergencia de vandalismo, que se vuelve cotidiano en un territorio donde más del 50\% de las viviendas son abandonadas a solo una década después de haberse entregado.

Considerando que los vínculos sociales constituyen el capital social de todo individuo, y siguiendo a Kaztman (2000), cuando habla del capital social de una persona y afirma que se trata de "su capacidad para movilizar la voluntad de otras personas en su beneficio sin recurrir a la fuerza o a la amenaza de fuerza", el capital social podría entenderse a partir de tres principales elementos: 1) la relación de obligaciones y derechos que genera una persona para recibir ayuda de otras, 2) la intensidad de los vínculos sociales y 3) el valor que las redes sociales le dan a los recursos para facilitar los logros de los miembros.

Desde esta perspectiva, uno de los principales problemas observado en zonas de precariedad urbana como Lomas del Sur es la desorganización social que caracteriza estos nuevos desarrollos urbanos. Tanto las débiles estructuras de seguridad social que ocasionan una mayor exposición frente a los riesgos como la poca temporalidad en la constitución del vecindario y la diversidad sociocultural en la procedencia hacen difícil la constitución de redes de apoyo entre los habitantes. 
Más aún, hay un proceso constante de descalificación entre los miembros de la zona urbana que suelen culparse unos a otros por el deterioro del espacio donde habitan. En este sentido, cabe pensar en los análisis de Paugam (1991), sobre la "descalificación social", en los que expone la representación social de la pobreza desde los efectos que causan la situación de fragilidad por exclusión de las estructuras del empleo, el asistencialismo y dependencia social y la ruptura de los vínculos sociales.

\section{Las consecuencias: informalidad, insalubridad e injusticia espacial}

La fragilidad no implica únicamente un estado temporal, sino que se reproduce a lo largo de la vida como condicionante que limita las oportunidades de movilidad social. Si se considera, por ejemplo, la débil infraestructura de transporte de Lomas del Sur y la necesidad de la población de desplazarse todos los días hasta Guadalajara para ir a trabajar, se puede entender cómo los habitantes, que invierten hasta cinco horas diarias en desplazamientos, pierden las posibilidades de realización personal y social que se podrían desarrollar en estos momentos. Por otro lado, la insuficiencia de espacios educativos, así como la falta de servicios de salud y de abasto de alimentos condicionan la vida ordinaria de todos los hogares de una manera más fuerte que quienes viven en fraccionamientos similares, pero dentro de la mancha urbana de Guadalajara.

Las resultantes de la fragilidad urbana que se manifiesta tanto en la morfología como en la carencia de infraestructura y servicios públicos en Lomas del Sur derivan en problemas como la informalidad y la inseguridad. De hecho, muchas de las viviendas que fueron abandonadas por sus propietarios originales que dejaron de pagar el crédito han sido hoy vandalizadas o invadidas por familias que se establecen en ellas a sabiendas de que no son suyas y que nunca tendrán un comprobante válido del domicilio que les acredite. Otras casas han sido invadidas para criar perros, para entrenar box o permanecen llenas de hierbas y basura porque nadie se les acerca. La inseguridad, en medio de estos paisajes decadentes, es un problema de todos los días, pero que se conjuga con falta de alumbrado público y la presencia de animales callejeros.

Las consecuencias de la fragilidad urbana, por lo tanto, se tejen con la vulnerabilidad social y aumentan la exposición de los individuos frente a las catástrofes que afectan a los territorios como las inundaciones, los terremotos y sequías. Las desventajas de los habitantes de zonas urbanas de precariedad, como los fraccionamientos, se van acumulando poco a poco y las amenazas también se multiplican. De esta manera, evidentemente, la afectación de los desastres tiene un componente sociourbano que debe ser estudiado desde las dinámicas sociales y las configuraciones del espacio. 


\section{Conclusiones}

Considerando la fragilidad como el componente físico y visible con el que se presentan las ciudades, que además se refuerza con la calidad de los vínculos sociales, la exposición de los habitantes urbanos frente a los fenómenos naturales está condicionada por una serie de factores relacionados con la configuración del espacio y con la organización de la sociedad. En los fraccionamientos populares donde habitan los grupos sociales de mayor precariedad, las catástrofes ocurren con mayor intensidad porque se van acumulando diversos problemas que aumentan el riesgo y debilitan las redes de protección social.

Por otro lado, es importante hacer notar la importancia de las estrategias del urbanismo para prevenir los desastres y mitigar los riesgos socialmente producidos, pero que se viven de manera individualizada a partir de las condiciones físicas sociales de cada persona. La armonización de políticas urbanas con la protección social podría reforzar las estrategias familiares y fraternas con que se suele responder ante las situaciones desastrosas, de tal manera que se genere una plataforma de resistencia que facilite a los habitantes cuando deben sobreponerse a un evento azaroso.

Es fundamental insistir en los abordajes socioespaciales de los riesgos que se viven en las ciudades y en la revisión constante de los conceptos con los que se piensa y se actúa en el mundo contemporáneo. Desde este análisis se evidencia la importancia de incluir los aspectos territoriales en la comprensión de la fragilidad y la vulnerabilidad humana que queda expuesta frente a los peligros, como inundaciones o terremotos, que exhiben las carencias de los sistemas urbanos y políticos para proteger a la población.

\section{Referencias}

Agier, M. (2015). Anthropologie de la ville. París: PUF.

Althabe, G. (2008). Entrevista. En T. Paquot, Conversations sur la ville et l'urbain avec Thierry Paquot. Lonrai, Francia: infolio.

Bayón, M. C. (2015). La integración excluyente: experiencias, discursos y representaciones de la pobreza urbana en México. México: UNAM/Bonilla Artigas.

Beck, U. (2008). La sociedad del riesgo mundial: En busca de la seguridad perdida. Barcelona: Paidós.

Bourdin, A. (2016). La métropole fragile. París: Le Moniteur.

Castel, R. (2006). La inseguridad social ¿Qué es estar protegido? Buenos Aires: El Manantial.

Colegio de Contadores Públicos de México (2016). El outsourcing en México: Pasado, presente y ifuturo? Puntos finos. Recuperado de: https://www.ccpm.org.mx/avisos/El\%20-outsourcing-\%20en\%20-mexiconoviembre-2016-puntos-finos.pdf

Gobierno de Tlajomulco (2016). Tlajomulco se mantiene en contingencia por inundaciones. Recuperado de: https:/ / tlajomulco.gob.mx/ prensa/tlajomulco-se-mantiene-en-contingencia-por-inundaciones

Gobierno de Tlajomulco (2014). Información fundamental licencias de urbanización agosto 2014. Recuperado de: https:/ / tlajomulco.gob.mx/sites/default/files/transparencia/licenciascomodatos/8Vtlicenciasdeurb anizacionagosto2014.pdf

Harvey, D. (2008). Géographie de la domination. París: Les Prairies Ordinaires. 
Katzman, R. (2000). Notas sobre la medición de la vulnerabilidad social. Serie Documentos de Trabajo del IPES / Colección Aportes Conceptuales No2. Santiago de Chile: CEPAL.

KOSMOS (2018). Beyond Urban Transformation: Interdisciplinary Perspectives on Urban Everyday Life. Berlín: Humboldt-University of Berlin. Recuperado de: https://issuu.com/carolin.genz/docs/beyondurban-transformation_2018

Martin, C. (2013). Penser la vulnérabilité. Les apports de Robert Castel. Alter: European Journal of Disability Research / Revue européenne de recherche sur le handicap, 7(4), 293-298. Recuperado de: https://halshs.archives-ouvertes.fr/halshs-00915581/document

Moser, C. (1998). Reassessing Urban Poverty Reduction Strategies: The Asset Vulnerability Framework. World Development, 26(1): 1-19. https:/ / doi.org/10.1016/S0305-750X(97)10015-8

Musset, A. (dir.). (2010). Ciudad, sociedad, justicia: un enfoque espacial y cultural. Mar del Plata: Eudem/Universidad Nacional de Mar del Plata.

Paugam, S. (1991). La disqualification sociale. Essai sur la nouvelle pauvreté. París: PUF.

Rawls, J., (1971). Teoría de la Justicia. México: Fondo de Cultura Económica.

Ríos-Llamas, C. (2018). Ciudades obesogénicas y mujeres vulnerables. Guadalajara: ITESO.

Schröder-Butterfill, E. y Marianti, R. (2006). A framework for understanding old-age vulnerabilities. Ageing $\mathcal{E}$ Society, 26, 9-35. doi:10.1017/S0144686X05004423

Sen, A. (1995). Nuevo examen de la desigualdad. Madrid: Alianza.

Torres, Raúl (30 de junio de 2019). Granizada deja más de 450 casas dañadas en Jalisco. El Universal. Recuperado de: https://www.eluniversal.com.mx/estados/granizada-deja-mas-de-450-casas-danadas-enguadalajara

Wacquant, L. (2007). Parias urbains. Ghetto, banlieues, Etat. Une sociologie comparée de la marginalité sociale. París: La Découverte.

Young I. M. (1990). Justice and the Politics of Difference. Princeton: Princeton University Press. 